\title{
Sensibilidad a la ansiedad y presión psicológica: Efectos sobre el rendimiento deportivo en adolescentes
}

\author{
Anxiety sensitivity and choking under pressure: Effects \\ on sport performance in adolescents
}

\section{Sensibilidade à ansiedade e pressão psicologica: Efeitos no desempenho esportivo em adolescentes}

\author{
José Molina, Bonifacio Sandín y Paloma Chorot
}

Facultad de Psicología, Universidad Nacional de Educación a Distancia, Madrid

\begin{abstract}
Resumen: El objetivo del presente estudio consistió en examinar la sensibilidad a la ansiedad y la afectividad positiva y negativa como posibles factores moduladores del choking (caída del rendimiento deportivo asociado a la presión psicológica). Participaron 100 estudiantes de secundaria (el 66\% eran mujeres), los cuales cumplimentaron el Childhood Anxiety Sensitivity Index (CASI) y el Positive and Negative Affect Schedule para Niños y Adolescentes (PANASN) antes de participar en un set de bádminton en condiciones de baja y alta presión. Los datos basados en ANOVAs apoyan la hipótesis de que la sensibilidad a la ansiedad, pero no la afectividad positiva o negativa, actúa como modulador del deterioro del rendimiento deportivo inducido por la condición de alta presión; los participantes con elevado nivel de sensibilidad a la ansiedad experimentaron una caída dramática en el rendimiento deportivo durante la condición de alta presión. El presente estudio amplía la literatura sobre el fenómeno del choking incluyendo la sensibilidad a la ansiedad como nueva variable relevante en el campo de la psicología de la actividad física y el deporte.

Palabras clave: Choking; rendimiento deportivo; sensibilidad a la ansiedad; afectividad; deporte; actividad física; adolescentes.

Abstract: The aim of the present study was to examine dispositional anxiety sensitivity and positive and negative affectivity as moderators of choking in sport. One hundred high school students (66\% were females) completed the Childhood Anxiety Sensitivity Index (CASI) and the Positive and Negative Affect Schedule for Children and Adolescents (PANASN) prior to completing a badminton set in low-pressure and high-pressure conditions.
\end{abstract}

A series of ANOVAs supported the hypothesis that anxiety sensitivity, but not positive or negative affectivity, acts as a moderator of the choking effect under psychological pressure; participants with high anxiety sensitivity showed a dramatic drop on sport performance during the high-pressured condition. The present study extends the choking literature including the anxiety sensitivity as a new relevant variable in this field of sport and physical activity psychology.

Keywords: Choking; anxiety sensitivity; affectivity; sport; physical activity; adolescents.

Resumo: O objetivo do presente estudo foi examinar a sensibilidade à ansiedade disposicional e afetividade positiva e negativa como moderadores de choking no esporte. Cem estudantes do ensino médio (66\% do sexo feminino) completaram a Ansiedade na Infância Índice de Sensibilidade (CASI) e do Afeto Positivo e Negativo Programação para Crianças e Adolescentes (PANASN) antes de completar um badminton definido em condiçôes de baixa pressão e de alta pressão. Os dados baseados em ANOVAS, apoiou a hipótese de que a sensibilidade à ansiedade, mas não afetividade positiva ou negativa, age como um moderador do deterioração esportivo sob pressão psicológica, os participantes com alta sensibilidade ansiedade mostrou uma queda dramática no desempenho esportivo durante a condição de alta pressão.

Palavras chave: choking, sensibilidade à ansiedade; afetividade; esporte, atividade física; adolescentes.

\section{Introducción}

La práctica del deporte puede implicar enfrentarse a situaciones donde la búsqueda del rendimiento deportivo constituya un elemento de extrema relevancia. En el rendimiento deportivo pueden influir diferentes tipos de variables no necesaria-

Dirección para correspondencia [Correspodence address]:

José Molina: Cuerda, 69, 13160, Ciudad Real, España. E-mail: jmolinadotor@yahoo.es

Bonifacio Sandín: UNED, Facultad de Psicología, Juan del Rosal 10 28040 Madrid, España. bsandin@psi.uned.es

Paloma Chorot: UNED, Facultad de Psicología, Juan del Rosal 10, 28040 Madrid, Espańa.pchorot@psi.uned.es mente de tipo físico, tales como la motivación, la atención, el estrés, la ansiedad, o la autoconfianza (Auweele, De-Cuyper, Van-Mele, y Rzewnicky, 1993; Buceta, 1990, 1996; García, Rodríguez, Andrade y Arce, 2006; Gil, Capafons y Labrador, 1993; Mahoney, 1989). Por ello, el conocimiento y la manipulación de algunas de estas variables, puede resultar de gran relevancia para la obtención de los mejores resultados deportivos.

Habitualmente en este tipo de situaciones, donde se busca el rendimiento como vía para superar al rival o a sí mismo, suele aparecer el fenómeno de "presión psicológica" sobre el 
deportista, el cual puede llegar a mermar sus facultades de cara a la competición y el rendimiento. Este concepto es denominado en la literatura científica como "choking under pressure" o "asfixia bajo presión", y aunque es un término muy utilizado, una definición unitaria sobre el mismo aún no ha sido definitivamente delimitada (Hill, Hanton, Mattews, Fleming, 2010). Aunque en el campo de la psicología del deporte se han utilizado, de forma más o menos indiferenciada, los conceptos de activación (o arousal), estrés y ansiedad, cuando se trata de competiciones deportivas, donde se busca obtener un rendimiento óptimo, se ha propuesto como más relevante el concepto de "presión psicológica deportiva" que, aunque puede incluir aspectos de los tres conceptos anteriores, puede aplicarse de forma más específica al ámbito deportivo.

Baumeister (1984) definió el choking under pressure ("asfixia bajo presión”) como una expresión metafórica usada para describir la ocurrencia de una reducción del rendimiento deportivo a pesar de los esfuerzos del individuo por conseguir un rendimiento superior. La presión (pressure) se ha definido como un factor o combinación de factores que incrementan la demanda de la situación (mayor importancia por conseguir un elevado rendimiento). El choking se refiere a la reducción del rendimiento bajo las situaciones de presión. El "ahogamiento" o asfixia bajo presión se ha asociado generalmente al ámbito de la competición deportiva, ya que la competición constituye en sí misma una forma de presión, la cual suele asociarse a incrementos significativos de los niveles de activación (Baumeister, 1984).

Hall (2002) redefinió el choking como una elevación en los niveles de ansiedad y activación bajo presión percibida, que conduce a un deterioro crítico en la ejecución habitual del rendimiento. Este concepto de presión psicológica en el deportista, choking o asfixia bajo presión (Hill et al. 2010), ha sido interpretado como un grave deterioro en la ejecución de los procesos habituales, como resultado de una elevación en la ansiedad bajo la presión psicológica percibida, lo cual puede llevar a reducciones significativas en el desempeño (Mesagno, Marchant y Morris, 2008). Este fenómeno se ha asociado, y a veces se ha identificado, con otros conceptos más clásicos como la ansiedad o el estrés (véase Gould y Krane, 1992; Weinberg y Gould, 1996).

Aunque inicialmente la psicología del deporte se interesó y centró fundamentalmente en el concepto de arousal, la investigación más reciente ha puesto más énfasis en los constructos de ansiedad y estrés (Woodman y Hardy, 2001). Una distinción importante entre la activación/arousal y la ansiedad es que la ansiedad implica la interpretación de la situación como una amenaza, mientras que la activación como tal no implica necesariamente tal interpretación (Hammermeister y Burton, 2001). Por otra parte, la ansiedad se ha sugerido como un mejor predictor de los resultados de rendimiento que el arousal cuando las tareas son de una naturaleza más compleja y con- tienen una mayor carga cognitiva (Arent y Landers, 2003).

Sobre la base de estos conceptos (arousal, activación, ansiedad y/o estrés), se han venido estableciendo diversas hipótesis relacionadas con el rendimiento deportivo, tales como la teoría de la inversión (Kerr, 1985), la teoría de la catástrofe (Hardy, 1990), la teoría del pensamiento eficiente (Eysenck y Calvo, 1992), la teoría del control atencional (Eysenck, Derakshan, Santos y Calvo, 2007) y la teoría del procesamiento consciente o de autofocalización (Beilock y Carr, 2001). Todas estas teorías enfatizan de algún modo el papel del individuo en relación con la percepción de sí mismo o de la situación en el contexto deportivo, como por ejemplo la interpretación particular (positiva o negativa) del nivel de arousal (teoría de la inversión), la preocupación o ansiedad (teorías de la catástrofe y del procesamiento eficiente), y los niveles de conciencia de la actividad (teoría del procesamiento consciente). Estas teorías, no obstante, únicamente han proporcionado puntos de vista parciales sobre la implicación de los procesos psicológicos en el rendimiento deportivo.

Aunque en general la psicología del deporte ha valorado la relevancia de diferentes manifestaciones o formas de la ansiedad, tales como las dimensiones cognitiva y somática (Jones, 1995; Woodman y Hardy, 2001), existen algunos constructos psicológicos relacionados con la ansiedad que podrían desempeñar un papel más relevante en este sentido, tal como ocurre con el concepto de sensibilidad a la ansiedad. La sensibilidad a la ansiedad ha sido definida como el miedo a los síntomas de ansiedad, el cual puede ocurrir por diversas causas, como la creencia de que las sensaciones de ansiedad poseen consecuencias peligrosas o dañinas (Reiss y McNally, 1985). La sensibilidad a la ansiedad no debe confundirse con el rasgo de ansiedad (Taylor, Koch y Crockett, 2001). Una persona puede tener un elevado rasgo de ansiedad (es decir, tendencia a experimentar ansiedad ante las situaciones estresantes) y, en cambio, poseer un nivel bajo de sensibilidad a la ansiedad (i.e., no reaccionar con miedo o ansiedad al experimentar los síntomas de ansiedad). La sensibilidad a la ansiedad tiene la peculiaridad de ser un "miedo fundamental", ya que puede motivar o amplificar cualquier otro tipo de miedo.

La sensibilidad a la ansiedad, al referirse al temor a los síntomas de ansiedad, suele implicar en gran medida a los síntomas fisiológicos de la ansiedad, tales como la taquicardia, las palpitaciones, la sensación de mareo, la respiración dificultosa o disnea, el temblor, etc. En este sentido, la actividad física suele inducir de forma natural gran parte de estos síntomas, lo cual puede ocurrir de forma más marcada cuando se trata de actividad física asociada a deportes de competición. Cabe afirmar, así mismo, que las personas con mayor grado de sensibilidad a la ansiedad tiendan a evitar ciertas actividades físicas debido al malestar que le produce la percepción de los síntomas fisiológicos producidos por el ejercicio físico, los cuales son similares a los que se producen asociados a la ansiedad. 
Recientemente, y procedente fundamentalmente de la investigación relacionada con el trastorno de pánico, se ha venido proponiendo el concepto de "evitación interoceptiva" para referirse a la evitación de este tipo de síntomas generados por diversos estímulos o situaciones que propician la activación de dichas sensaciones (Sandín, 2005; White y Barlow, 2002). La evitación interoceptiva puede ser muy sutil y mucho menos marcada que la que se produce en los individuos agorafóbicos, e incluye diversos tipos de actividades o situaciones más o menos heterogéneas que comparten la propiedad de estimular la función interoceptiva e inducir sensaciones somáticas, tales como correr (y en general practicar cualquier tipo de actividad física), tomar bebidas excitantes, etc. Todas ellas tienen en común que activan la función fisiológica e inducen síntomas somáticos similares a los que se producen asociados a los estados de hiperventilación y/o pánico.

La evitación interoceptiva puede perturbar de forma significativa el rendimiento deportivo, tal y como ha sido señalado en algunos trabajos recientes (p.ej., Gimeno y Ezquerro, 2006). Estos autores han desarrollado un programa de intervención de 8 sesiones para reducir el miedo a los síntomas y la evitación interoceptiva asociadas al rendimiento deportivo (fue aplicado con éxito por los autores a un caso de excesiva evitación interoceptiva ante una prueba de 1.500 metros en pista, en el contexto de una oposición para acceder a una plaza de policía local).

Se ha sugerido que la evitación interoceptiva se asocia a niveles elevados de sensibilidad a la ansiedad (Taylor, 2000). La sensibilidad a la ansiedad potencia, por naturaleza, la evitación interoceptiva, ya que tal evitación se produce por el temor a los síntomas de ansiedad. Por otra parte, existe evidencia de que la sensibilidad a la ansiedad incrementa la percepción autónoma, i.e., la percepción de los síntomas somáticos de ansiedad (Taylor, 1999). Por tanto, cabría suponer que las personas con niveles elevados de sensibilidad a la ansiedad experimentarán reducciones significativas del rendimiento deportivo, especialmente en condiciones de alta presión psicológica (i.e., situaciones en las que el rendimiento es de gran relevancia para el deportista), ya que en dichas situaciones se incrementa la activación fisiológica y la percepción de los síntomas somáticos de la ansiedad.

El presente estudio tiene como finalidad probar la efectividad de un procedimiento de manipulación experimental de la presión psicológica deportiva en un contexto de rendimiento deportivo. Así mismo, pretendemos examinar la influencia de la sensibilidad a la ansiedad sobre el rendimiento deportivo en función del grado de presión psicológica. Para ello partimos de dos hipótesis fundamentales. De acuerdo con nuestra primera hipótesis, la condición de elevada presión psicológica deberá asociarse a menor rendimiento deportivo que la condición de baja presión psicológica (i.e., la condición de alta presión producirá un deterioro del rendimiento deportivo, en comparación con la condición de baja presión que no debería asociarse a dicho deterioro). Mediante la segunda hipótesis postulamos que la sensibilidad a la ansiedad debería modular el efecto que induce la manipulación experimental sobre el rendimiento, incrementando el deterioro del rendimiento deportivo únicamente bajo la condición de alta presión psicológica. Finalmente, y como tercera hipótesis, asumimos un efecto similar para el afecto negativo, aunque no para el afecto positivo, considerando que la afectividad negativa facilita el incremento de las reacciones de ansiedad en situaciones de estrés (i.e., bajo la condición de alta presión psicológica). Estos dos fenómenos asociados a las hipótesis segunda y tercera deberían darse tras controlar el efecto de la variable sexo.

\section{Método}

\section{Diseño}

Se estableció un diseño de dos condiciones experimentales de medidas repetidas. Las condiciones experimentales consistían en una condición de baja presión deportiva y una condición de alta presión deportiva (el diseño incluía también una fase previa de familiarización). Las condiciones correspondían a partidos de bádminton de un set. Todos los participantes participaron en ambas condiciones. (Véase la sección sobre el procedimiento).

\section{Participantes}

Se seleccionó una muestra de 100 alumnos del Instituto de Enseñanza Secundaria Mónico Sánchez (Piedrabuena, Ciudad Real), pertenecientes al curso académico 2011/2012. Los alumnos cursaban el nivel post-obligatorio de Bachillerato en cualquiera de las dos modalidades que oferta el centro educativo (Humanidades y Ciencias Sociales, Ciencias Naturales y Tecnológico). La muestra fue seleccionada al azar a partir de todos los alumnos de Bachillerato, entre los 15 y 17 ańos, del citado centro educativo. La muestra estaba constituida por 44 varones y 66 mujeres, y estaba aproximadamente equiparada en edad entre varones y mujeres $(\mathrm{M}=16.1, \mathrm{DT}=.55)$.

\section{Instrumentos de evaluación}

Previo a la aplicación de las condiciones experimentales, los participantes cumplimentaron los siguientes cuestionarios de autoinforme de forma individual durante una sesión en el aula en horario de clase.

Cuestionario PANAS de Afecto Positivo y Negativo para Niños y Adolescentes (PANASN; Sandín, 1997). Prueba elaborada por Sandín $(1997,2003)$ para niños y adolescentes a partir de la forma para adultos del Positive and Negative Affect Schedule (PANAS) de Watson, Clark y Tellegen (1988; 
Sandín et al., 1999). El PANASN, al igual que el PANAS, es un cuestionario de autoinforme de 20 elementos. Diez items evalúan el afecto positivo (p.ej., "Soy una persona animada, suelo emocionarme") y otros diez el afecto negativo (p.ej., "Me siento nervioso"). El cuestionario es cumplimentado por el niño/adolescente teniendo en cuenta la manera en que éste se siente y/o comporta habitualmente, siguiendo una escala de tres alternativas de respuesta, descritas éstas como "Nunca" (1), "A veces" (2), y "Muchas veces" (3).

Childhood Anxiety Sensitivity Index (CASI; Silverman, Fleisig, Rabian y Peterson, 1991; version española de Sandín, Chorot, Santed, y Valiente, 2002). Consta de 18 items dirigidos a evaluar la sensibilidad a la ansiedad en población de nińos y adolescentes. Los ítems se refieren a consecuencias físicas, cognitivas y sociales de la ansiedad. El cuestionario se contesta según una escala Likert de 3 alternativas: Nada (1), Un poco (2), Mucho (3). Se obtiene una puntuación total en sensibilidad a la ansiedad sumando las puntuaciones obtenidas en los 18 ítems. Datos sobre la fiabilidad y validez de la versión española de la CASI fueron referidos a partir de la validación llevada a cabo por Sandín et al. (2002).

\section{Definición de las variables de rendimiento}

Durante las tres fases del experimento (fase de familiarización, fase de competición sin presión y fase de competición con presión) se evaluó la práctica deportiva de bádminton del participante a través de hojas de registro de observaciones, donde se registraron los valores individuales de cada uno de los participantes para las dos variables siguientes:

Errores no forzados (ENF) por el rival. Esta variable de rendimiento deportivo indica los errores que un jugador comete independientemente de la acción motriz del rival.

Puntos conseguidos (PC). Puntos que cada jugador consigue dentro de un set. Variable dependiente que resume las acciones motrices que conllevan a conseguir un tanto o punto.

Los registros fueron realizados por el profesor de la asignatura de Educación Física. Con objeto de probar la fiabilidad de los registros se calculó el grado de concordancia entre dos jueces (el profesor de la asignatura y otro profesor entrenado) que evaluaron 20 casos, seleccionados de manera aleatoria, obteniéndose un coeficiente kappa de Cohen de 98.

\section{Procedimiento}

El procedimiento se llevó a cabo en las cuatro fases, que incluían la aplicación de los cuestionarios de autoinforme, una fase de familiarización con el juego, y dos fases de competición. La duración del juego (set) durante las fases de competición fue de aproximadamente $25 \mathrm{~min}$. (cada juego concluía cuando una de las parejas obtenía 21 puntos).

Una primera fase (fase de aplicación de los cuestionarios de autoinforme) se utilizó para informar a los alumnos sobre el trabajo de investigación y obtener su consentimiento informado. Así mismo, durante esta fase se aplicaron los cuestionarios de autoinforme PANASN y CASI.

La segunda fase, previamente a la intervención, fue la fase de familiarización con la actividad deportiva del bádminton (i.e., situación de preparación y entrenamiento). En esta fase, que tuvo una duración de 15 sesiones de 55 minutos cada una, se inició la unidad didáctica de bádminton, explicando las características del juego, el reglamento, algo de historia, los golpes técnicos más relevantes, las situaciones tácticas más influyentes y la dinámica del juego. Posteriormente, se realizaron ejercicios prácticos y actividades de juego de bádminton con la finalidad que el alumno adquiriese una experiencia mínima, incluidas la ejecución de golpes y las tácticas. Al final de esta etapa se practicó en las situaciones de partido de modalidad individual y de dobles. El estudio (fases tercera y cuarta) se llevó a cabo en la modalidad de dobles.

En la tercera fase, que correspondió a la fase de intervención sin presión (i.e., situación de competición de baja presión deportiva), las parejas participaron en una situación real de partido a un set de bádminton, fuera de las horas de clase. Se explicó a los participantes que se trataba de una situación de competición sin evaluación (con la finalidad de reducir la presión deportiva), que serviría para preparar la situación posterior de competición con evaluación (i.e., fase con presión deportiva). Durante esta tercera fase los participantes jugaron un set de dobles de bádminton en condiciones de baja presión deportiva. Para conseguir este tipo de situación se eliminaron los elementos que habitualmente suelen incrementar el estrés deportivo y/o la ansiedad competitiva, tales como la asistencia de público, la percepción de ser grabado, y el que la práctica realizada influya en la calificación del alumno. Se realizó el registro durante el juego, cuantificándose los golpes ganadores y puntos conseguidos en el set.

Finalmente, la cuarta fase correspondió a la fase de intervención con presión (i.e., situación de competición de alta presión deportiva) y se llevó a cabo bajo las siguientes condiciones que supuestamente deberían incrementar el nivel de presión deportiva: (a) Las sesiones se llevaron a cabo durante las horas oficiales de clase de la asignatura de Educación Física. (b) El profesor grabó con una cámara fija con trípode el desempeńo de cada pareja; los participantes sabían que estaban siendo grabados. (c) El profesor registraba el desempeño de los participantes, habiéndose informado que el resultado del partido influiría en la calificación de la evaluación de la asignatura de Educación Física. (d) El resto de alumnos de la clase observaron y registraron los desempeños, con la finalidad de aumentar la presión psicológica sobre los participantes al verse estos últimos evaluados por sus compañeros. Además, a la pareja ganadora se le subían dos puntos (20\%) en la calificación final de la asignatura de Educación Física. 


\section{Resultados}

En la Tabla 1 se indican los valores (medias y DTs) obtenidos para las variables de afectividad (afecto positivo y afecto negativo) y sensibilidad a la ansiedad en función del género. Se aplicó un diseño de análisis de varianza (ANOVA) de un factor (varón vs. mujer) para cada una de las tres variables de diferencias individuales. Así mismo, en dicha tabla se indican los coeficientes de consistencia interna (alfa de Cronbach) relativos a estas tres variables. Como puede apreciarse, encontramos diferencias significativas entre varones y mujeres en afectividad negativa (PANAS-N) aunque, como indica el valor de eta ${ }^{2}$ parcial, el significado de esta diferencia es mínimo. Los valores de consistencia interna fueron elevados (i.e., superiores a .80) para las tres medidas de diferencias individuales.

Tabla 1. Medias y desviaciones típicas para las variables de diferencias individuales (afecto positivo, afecto negativo, y sensibilidad a la ansiedad) según género.

\begin{tabular}{llllllll}
\hline & \multicolumn{3}{c}{ Varones } & \multicolumn{3}{c}{ Mujeres } \\
\cline { 2 - 5 } & Media & $D T$ & Media & $D T$ & $F(1,98)$ & Eta ${ }^{2}$ parcial & $\alpha$ \\
PANASN-P & 23,41 & 3,11 & 22,86 & 3,06 & 0,79 & 0,01 & 0,82 \\
PANASN-N & 18,64 & 2,60 & 20,00 & 2,82 & $6,17^{*}$ & 0,06 & 0,81 \\
CASI & 33,52 & 7,97 & 32,75 & 7,35 & 0,25 & 0,01 & 0,89 \\
\hline Nota: PANASN $=$ Positive and Negative Affect Scale para Niños y Adolescentes (P = Afecto positivo; N = Afecto negativo). \\
CASI = Childhood Anxiety Sensitivity Index. ${ }^{*}$ p<.05.
\end{tabular}

Efectos de las condiciones experimentales sobre el rendimiento deportivo

Los resultados confirman la efectividad del procedimiento experimental sobre el rendimiento deportivo (véase la Tabla 2). Como puede apreciarse en dicha tabla, se produce un incremento de errores no forzados y un decremento de puntos conseguidos en la condición de alta presión, en comparación con las puntuaciones obtenidas en la condición de baja presión. Tras aplicar un diseño de ANOVA de medidas repeti- das de un factor (baja presión vs. alta presión) encontramos diferencias significativas entre ambos niveles de la condición experimental tanto para los errores no forzados como para los puntos conseguidos. Así mismo, puede observarse que la condición experimental explica una proporción importante de la varianza de ambas variables dependientes (errores no forzados y puntos conseguidos). Como se indica en la tabla, el rendimiento deportivo es significativamente mayor en el nivel de baja presión que en el de alta presión.

Tabla 2. Medias y desviaciones típicas en rendimiento deportivo (errores no forzados y puntos conseguidos) en función del grado de presión deportiva (baja vs. alta).

\begin{tabular}{|c|c|c|c|c|c|c|}
\hline & \multicolumn{2}{|c|}{ Baja presión } & \multicolumn{2}{|c|}{ Alta presión } & \multirow[b]{2}{*}{$F(1,99)$} & \multirow[b]{2}{*}{$\mathrm{Eta}^{2}$ parcial } \\
\hline & Media & $D T$ & Media & $D T$ & & \\
\hline Errores no forzados & 3.41 & 1.85 & 5.48 & 2.60 & $78.77^{* * *}$ & .443 \\
\hline Puntos conseguidos & 8.17 & 3.10 & 6.56 & 3.45 & $27.78^{* * *}$ & 219 \\
\hline
\end{tabular}

Efectos moduladores de las variables individuales sobre el rendimiento deportivo

Con objeto de examinar el posible efecto modulador de las variables de diferencias individuales (sensibilidad a la ansiedad, afecto positivo y afecto negativo) aplicamos 3 diseńos de análisis de covarianza de $2 \times 2$ para cada una de las dos variables dependientes (errores no forzados y puntos conseguidos), controlando el efecto de la variable sexo. En todos los diseńos, la primera variable independiente consistió en la condición experimental "presión deportiva" (baja presión vs. alta presión) y la segunda era una de las tres variables de diferencias individuales (sensibilidad a la ansiedad, afecto positivo, o afecto negativo). Para ello, estas variables se recodificaron en valores bajo vs. alto según que puntuaran por debajo o por encima de la media. Los resultados de estos análisis, así como las medias y DTs, aparecen en las Tablas 3 y 4. 
Tabla 3. Medias y DTs en errores no forzados en función de la condición experimental (baja vs. alta presión) y las variables de diferencias individuales (PANASN-P, PANASN-N, y CASI) (nivel bajo vs. alto).

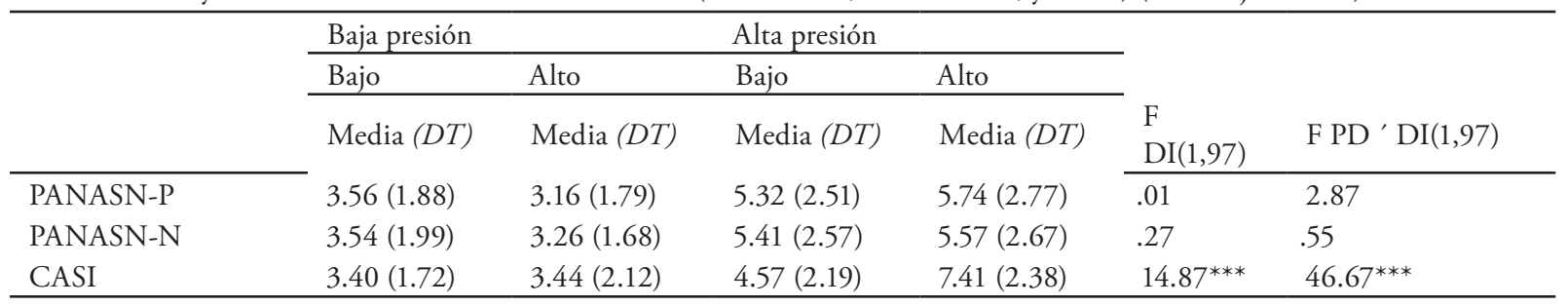

Nota. PANASN = Positive and Negative Affect Scale para Niños y Adolescentes ( $\mathrm{P}=$ Afecto positivo; $\mathrm{N}=$ Afecto negativo). CASI = Childhood Anxiety Sensitivity Index. F DI = valor de F para el factor de diferencias individuales. F PD ' DI = valor de F para la interacción entre los factores de presión deportiva y diferencias individuales. ${ }^{* * *} \mathrm{p}<.001$.

Tabla 4. Medias y DTs en puntos conseguidos en función de la condición experimental (baja vs. alta presión) y las variables de diferencias individuales (PANASN-P, PANASN-N, y CASI) (nivel bajo vs. alto).

\begin{tabular}{llllllll}
\hline & \multicolumn{3}{l}{ Baja Presión } & \multicolumn{2}{l}{ Alta presión } & & \\
\cline { 2 - 5 } & Bajo & Alto & Bajo & Alto & & \\
\cline { 2 - 5 } & Media $(D T)$ & Media $(D T)$ & Media $(D T)$ & Media $(D T)$ & & F DI(1,97) & F PD ' DI(1,97) \\
\cline { 2 - 5 } PANASN-P & $8.21(3.27)$ & $8.11(2.86)$ & $7.00(3.52)$ & $5.84(3.24)$ & & 1.06 & 2.75 \\
PANASN-N & $9.06(3.29)$ & $7.13(2.53)$ & $7.46(3.48)$ & $5.50(3.12)$ & & $11.64^{* *}$ & .02 \\
CASI & $8.43(3.28)$ & $7.63(2.67)$ & $7.96(3.16)$ & $3.59(1.72)$ & & $21.05^{* * *}$ & $43.46^{* * *}$ \\
\hline
\end{tabular}

Nota. PANASN = Positive and Negative Affect Scale para Niños y Adolescentes ( $\mathrm{P}=$ Afecto positivo; $\mathrm{N}=$ Afecto negativo). CASI = Childhood Anxiety

Sensitivity Index. F DI = valor de F para el factor de diferencias individuales. F PD ' DI = valor de F para la interacción entre los factores de presión deportiva y diferencias individuales. ${ }^{* *} \mathrm{p}<.01 ;{ }^{* * *} \mathrm{p}<.001$.

En la Tabla 3 indicamos los datos relativos a la variable dependiente errores no forzados. Como puede apreciarse, en general los errores se asocian en mayor medida a la condición de alta presión que a la de baja presión. En relación con los efectos de las variables de diferencias individuales, únicamente resultó significativo el efecto de la sensibilidad a la ansiedad (CASI; $\mathrm{p}<.001$ ), no siendo significativos los efectos de la afectividad negativa ni de la afectividad positiva. Es importante señalar que también fue significativa la interacción entre la sensibilidad a la ansiedad y la condición experimental ( $\mathrm{p}<.001)$.

Con objeto de examinar de forma más específica esta interacción, indicamos en la Figura 1 los valores medios en errores no forzados en función de los niveles de ambos factores (i.e., sensibilidad a la ansiedad alta vs. baja y presión deportiva alta vs. baja). Tal y como puede apreciarse en la figura, la mayor presión deportiva (condición de alta presión) incrementa el número de errores, si bien este incremento es dramáticamente mayor para la condición de elevada sensibilidad a la ansiedad. Dicho en otros términos, la sensibilidad a la ansiedad tiende a modular el efecto de deterioro del rendimiento que induce la presión deportiva facilitando la ocurrencia de errores no forzados.
Figura 1. Representación gráfica de la interacción entre la sensibilidad a la ansiedad y la presión deportiva, para la variable dependiente errores no forzados. CASI = Childhood Anxiety Sensitivity Index.

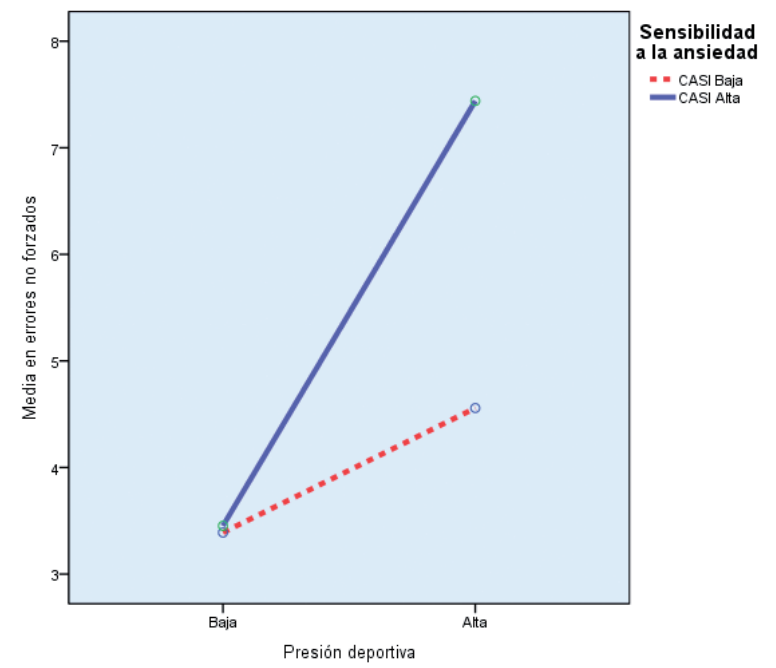

En relación con la variable dependiente puntos conseguidos, procedimos de forma similar a lo referido para los errores no 
forzados; los resultados se indican en la Tabla 4. Tal y como se refleja en la tabla, en general suelen obtenerse mejores puntuaciones (más puntos conseguidos) en la condición de baja presión deportiva que en la condición de alta presión. Tras la aplicación de los análisis de varianza constatamos efectos significativos de los factores de personalidad afecto negativo (PANASN-N) y sensibilidad a la ansiedad (CASI), no resultando significativo el efecto de la variable de personalidad afecto positivo (PANAS-P). Ambas variables de personalidad se asocian negativamente al rendimiento deportivo; en general los puntos conseguidos tienden a reducirse en los sujetos pertenecientes a los grupos correspondientes a los niveles elevados de sensibilidad a la ansiedad o afectividad negativa.

$\mathrm{Al}$ igual que para la variable dependiente errores no forzados, calculamos las posibles efectos de interacción de las variables independientes, resultando significativa únicamente la interacción entre la sensibilidad a la ansiedad y la presión deportiva (en la Figura 2 indicamos los efectos de interacción entre estas dos variables sobre la variable dependiente puntos conseguidos). Como puede observarse en la figura, los puntos conseguidos tienden a reducirse en la condición de alta presión deportiva; no obstante, la reducción resulta particularmente dramática en los participantes con elevado nivel de sensibilidad a la ansiedad (los individuos con bajo nivel de sensibilidad a la ansiedad apenas experimentan cambio). Así mismo, se observa que, aunque los participantes con elevado nivel de sensibilidad a la ansiedad suelen obtener peores puntuaciones que los participantes con niveles bajos, la diferencia es mucho más marcada en la condición de alta presión deportiva.

Figura 2. Representación gráfica de la interacción entre la sensibilidad a la ansiedad y la presión deportiva, para la variable dependiente puntos conseguidos. CASI = Childhood Anxiety Sensitivity Index.

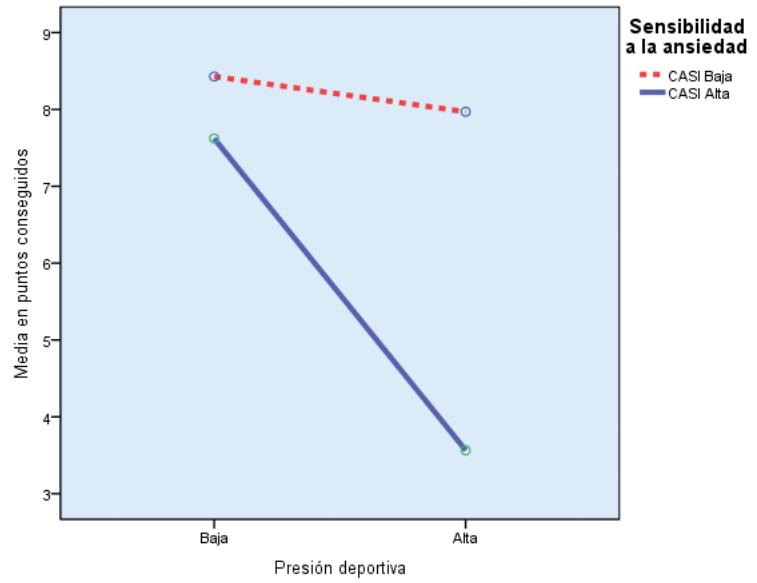

\section{Discusión}

Dos aspectos centrales han guiado la presente investigación. Por una parte, tratábamos de elaborar un procedimiento experimental de inducción de presión psicológica aplicable a la investigación en el ámbito deportivo. Por otra parte, pretendíamos estudiar el posible papel modulador de la sensibilidad a la ansiedad sobre los efectos que posee la presión psicológica en el deporte de competición, y adicionalmente el efecto modulador del afecto negativo.

Un primer resultado destacable viene dado por la efectividad del procedimiento experimental de inducción de presión psicológica deportiva. Hemos constatado diferencias estadísticamente significativas en el rendimiento deportivo de competición entre la condición de baja presión y la condición de alta presión, siendo significativamente más bajo el rendimiento en la condición de alta presión deportiva. Este resultado es consistente con la evidencia de la literatura que ha asociado el deterioro del rendimiento deportivo a las condiciones de mayor presión (choking pressure) (p.ej., Hill et al., 2010; Mesagno et al., 2008). Por tanto, aparte de extender la evidencia sobre este fenómeno, mediante el presente estudio se proporciona un nuevo procedimiento o paradigma experimental para su investigación en el contexto del deporte de competición de bádminton.

El segundo objetivo de nuestra investigación se centraba en estudiar el posible papel de las variables de personalidad sobre el rendimiento deportivo. Más específicamente, habíamos hipotetizado que la sensibilidad a la ansiedad debería modular el efecto inducido sobre el rendimiento deportivo por la presión psicológica. En este sentido, nuestros datos confirman el papel modulador de la sensibilidad a la ansiedad, la cual incrementa significativamente el efecto de la presión psicológica (i.e., los efectos producidos por la condición de alta presión psicológica). Este resultado en consistente con datos previos sobre la importancia de la sensibilidad a la ansiedad como variable que posee la propiedad de amplificar las respuestas de miedo y ansiedad, y por su propia naturaleza de inducción de temor a los propios síntomas fisiológicos de ansiedad (p.ej., taquicardia, falta de aliento, temblor, etc.) (Taylor, 1999; Taylor et al., 2001). Cabe afirmar que estos síntomas de temor a los síntomas de ansiedad pueden interferir en el desempeño deportivo, tal y como se desprende de los resultados obtenidos en el presente estudio. La sensibilidad a la ansiedad, al amplificar los síntomas de ansiedad, puede incrementar los efectos de distracción o interferencia relacionados con la ansiedad (Eysenck y Calvo, 1992; Eysenck et al., 2007; Hill y Shaw, 2013; Marchant y Gibbs, 2004), lo cual podría explicar la marcada reducción en el rendimiento deportivo asociada a los niveles elevados de sensibilidad a la ansiedad.

Así mismo, la elevada sensibilidad a la ansiedad ha sido asociada incrementos de la evitación interoceptiva, fenóme- 
no éste característico en las personas con sintomatología de pánico (Sandín, 2005). La evitación interoceptiva puede constituir, por tanto, una variable relevante implicada en el efecto modulador observado en los participantes con elevada sensibilidad a la ansiedad, aspecto que deberá ser examinado en futuras investigaciones. La evitación interoceptiva puede interferir en el rendimiento deportivo que se lleve a cabo bajo elevada presión, reduciendo la ejecución de posibles conductas involucradas en el deporte de competición. Recientemente, Gimeno y Ezquerro (2006) constataron reducciones del rendimiento deportivo asociadas a la evitación interoceptiva, demostrando la efectividad de un programa dirigido a la reducción de la evitación interoceptiva.

No hemos constatado efectos moduladores para las variables del afecto. El afecto positivo resultó ser irrelevante en relación con el rendimiento deportivo. En contraste, el afecto negativo se asoció significativamente a un menor rendimiento deportivo en ambas condiciones (alta y baja presión deportiva). Dada la naturaleza de la afectividad negativa (véase Sandín et al., 1999; Watson et al., 1988), este último resultado es coherente con las hipótesis que han defendido cierto deterioro del rendimiento deportivo asociado a los mayores niveles de estrés y ansiedad personales (Woodman y Hardy, 2001).
Que nosotros sepamos, éste es el primer estudio en el que se investiga la influencia de la sensibilidad a la ansiedad sobre el rendimiento deportivo de competición bajo presión. Como novedad ańadida respecto de investigaciones anteriores destacamos que en este estudio el procedimiento se ha basado en situaciones reales de juego, y no sobre situaciones simplificadas o modificadas como en investigaciones anteriores; esto le concede al estudio un mayor valor a la hora de generalizar sus resultados. Aunque el estudio se ha focalizado en un deporte (bádminton) y en un contexto de moderada presión psicológica, en principio resulta coherente su generalización a otros deportes y a otras condiciones deportivas de mayor nivel competitivo y mayor presión psicológica, donde los efectos posiblemente sean aun más dramáticos. Por otra parte, nuestros resultados también pueden ser aplicables al desarrollo de la investigación y práctica del deporte de competición en los contextos educativos (aplicación en programas de formación y enseñanza del deporte) y clínicos (el deporte de competición puede constituir un contexto facilitador de trastornos psicológicos infantojuveniles asociados a las personas con elevado nivel de sensibilidad a la ansiedad, tales como los trastornos de ansiedad).

\section{Referencias}

1. Arent, S. M. y Landers, D. M. (2003). Arousal, anxiety, and performance: A reexamination of the inverted-U hypothesis. Research Quarterly for Exercise and Sport, 74, 436-444.

2. Auweele, Y. V., De-Cuyper, B., Van-Mele, V. y Rzewnicky, R. (1993). Elite Performance and Personality: From Description and Prediction to Diagnosis and Intervention. En R. N. Singer, M. Murphey, y L. K. Tennant (eds.), Handbook of Research on Sport Psychology (pp. 257-299). New York: Macmillan.

3. Baumeister, R. F. (1984). Choking under pressure: self-consciousness and paradoxical effects of incentives on skillful performance. Journal of Personality and Social Psychology, 46, 610-620.

4. Beilock, S. L. y Carr, T. H. (2001). On the fragility of skilled performance: What governs choking under pressure? Journal of Experimental Psychology: General, 130, 701-725.

5. Buceta, J. M. (1990). Aspectos a tener en cuenta en relación con las deportistas españolas de alta competición. Seminario Mujer y Deporte, organizado por el Consejo Superior de Deportes, la Secretaría de Estado de Educación y el Instituto de la Mujer en Madrid. Publicado posteriormente en Planificación del Entrenamiento Deportivo y Rendimiento: Un enfoque interdisciplinar. Málaga: Instituto Andaluz del Deporte, 1995.

6. Buceta, J. M. (1996). Psicología y lesiones deportivas: prevención y recuperación. Madrid. Dykinson.

7. Eysenck, M. V. y Calvo, M. (1992). Anxiety and performance: The processing efficiency theory. Cognition and Emotion, 6, 409-434.

8. Eysenck, M. W., Derakshan, N., Santos, R. y Calvo, M. G. (2007). Anxiety and cognitive performance: Attentional control thery. Emotion, 7, 336-353.

9. García, E. M., Rodríguez, M., Andrade, E. M. y Arce, C. (2006). Adaptación del cuestionario MSCI para la medida de la cohesión en futbolistas jóvenes españoles. Psicothema, 18, 668-672.

10. Gil, M., Capafons, B. y Labrador, E. (1993). Variables físicas y psico- lógicas predictoras del rendimiento deportivo y del cambio terapéutico. Psicothema, 5, 97-110.

11. Gimeno, F. y Ezquerro M. (2006). Intervención psicológica en un caso de evitación interoceptiva en el deporte. Revista de Psicopatología y Psicología Clinica, 11, 99-106.

12. Gould, D. y Krane, V. (1992). The arousal-athletic performance relationship: current status and future directions. En T. S. Horn (ed.) Advances in sport psychology (1a ed., pp. 119-141). Champaign: Human Kinetics.

13. Hall, E. (2002). Defining choking: A qualitative examination. Unpublished Masters thesis, Victoria University, Victoria, Australia.

14. Hammermeister, J. y Burton, D. (2001). Stress, appraisal, and coping revisited: Examining the antecedents of competitive state anxiety with endurance athletes. The Sport Psychologist, 15, 66-90.

15. Hardy, L. (1990). A catastrophe model of performance in sport. En J. G. Jones y L. Hardy (eds.), Stress and performance in sport (pp. 81-106). Chichester: Wiley.

16. Hill, D. M., Hanton, S., Matthews, N. y Fleming, S. (2010). Choking in sport: a review. International Review of Sport and Exercise Psychology, 3, 24-39.

17. Hill, D. M. y Shaw, G. (2013). A qualitative examination of choking under pressures in team sport. Psychology of Sport and Exercise, 14, 103-110.

18. Jones, G. (1995). More than just a game: Research developments and issues in competitive anxiety in sport. British Journal of Psychology, 86, 449-478.

19. Kerr, J. H. (1985). The experience of arousal: a new basis for studying arousal effects in sport. Journal of Sport Sciences, 3, 169-179.

20. Mahoney, M. J. (1989). Psychological predictors of elite and non-elite performance in olympic weightlifting. International Journal of Sport Psychology, 20, 1-12.

21. Mesagno, C., Marchant, D. y Morris, T. (2008). A pre-performance 
routine to alleviate choking in "choking-susceptible" athletes. The Sport Psychologist, 22, 439-457.

22. Reiss, S. y McNally, R. J. (1985). The expectancy model of fear. En S. Reiss y R. R. Bootzin (eds.), Theoretical issues in behavior therapy. (pp. 107-121). London: Academic Press.

23. Sandín, B. (1997). Ansiedad, miedos y fobias y niños y adolescentes. Madrid: Dykinson.

24. Sandín, B. (2003). Escalas PANAS de afecto positivo y negativo para niños y adolescentes (PANASN). Revista de Psicopatología y Psicología Clinica, 8, 173-182.

25. Sandín, B. (2005). Evitación interoceptiva: Nuevo constructo en el campo de los trastornos de ansiedad. Revista de Psicopatología y Psicología Clínica, 10, 103-114.

26. Sandín, B., Chorot P., Lostao L., Joiner T. E., Santed M. A. y Valiente R. M. (1999). Escalas PANAS de afecto positivo y negativo: validación factorial y convergencia transcultural. Psicothema, 11, 37-51.

27. Sandín, B., Chorot, P., Santed, M. A. y Valiente, R. M. (2002). Análisis factorial confirmatorio del Índice de Sensibilidad a la Ansiedad para Niños. Psicothema, 14, 333-339.

28. Silverman, W. K., Fleisig, W., Rabian, B. y Peterson, R. A. (1991). Childhood Anxiety Sensitivity Index. Journal of Clinical Child Psychology, 20, 162-168.
29. Taylor, S. (Ed.) (1999). Anxiety sensitivity: theory, research and treatment of the fear of anxiety. Mahwah, NJ: LEA.

30. Taylor, S. (2000). Understanding and treating panic disorder. New York: Wiley.

31. Taylor, S., Koch, W.J. y Crockett, D.J. (1991). Anxiety sensitivity, trait anxiety and the anxiety disorders. Journal of Anxiety Disorders, 5, $293-$ 311.

32. Wang, J., Marchant, D., Morris, T., y Gibbs, P. (2003). Self-consciousness and trait anxiety as predictors of choking in sport. Journal of Science Medicine \& Sport, 7, 174-185.

33. Watson, D., Clark, L.A., y Tellegen, A. (1988). Development and validation of brief measures of positive and negative affect: The PANAS scales. Journal of Personality and Social Psychology, 54, 1063-1070.

34. Weinberg, R. S. y Gould, D. (1996). Fundamentos de psicología del deporte y del ejercicio físico. Barcelona: Ariel.

35. Woodman, T. y Hardy, L. (2001). Stress and anxiety. En R. N. Singer, H. A. Hausenblas y M. Janelle (eds.), Handbook of sport psychology (2a ed.) (pp. 290-318). New York: John Wiley and Sons, Inc. 